\title{
Spongiform Encephalopathy
}

National Cancer Institute

\section{Source}

National Cancer Institute. Spongiform Encephalopathy. NCI Thesaurus. Code C27585.

Encephalopathy that is characterized by tiny holes apparent by microscopy, often due to prion disease. 